\title{
COUNTABLE CONNECTED SPACES
}

\section{WILLIAM GUSTIN}

Introduction. Let $\subseteq$ be the class of all countable and connected perfectly separable Hausdorff spaces containing more than one point. It is known that an $\widetilde{S}$-space cannot be regular or compact. Urysohn, using a complicated identification of points, has constructed the first example of an $\widetilde{5}$-space. ${ }^{1}$ Two $\widetilde{S}$-spaces, $X$ and $X^{*}$, more simply constructed and not involving identifications, are presented here. The space $X^{*}$ is a connected subspace of $X$ and contains a dispersion point; that is, the subspace formed from $X^{*}$ by removing this one point is totally disconnected.

1. Sequences. The null sequence or any finite sequence of positive integers will hereafter be called more briefly a sequence. The null sequence or a sequence having an even number of elements is said to be even and a sequence having an odd number of elements is said to be odd. A sequence will usually be denoted by a lower case Greek letter: an arbitrary sequence by $\alpha, \beta$, or $\gamma$; an arbitrary even sequence by $\lambda, \mu$, or $\nu$; the null sequence by $o$. A positive integer will be denoted by a lower case italic letter (not $x, y$, or $z$ ), which may also serve to represent the sequence consisting of that single integer.

The relation $\alpha \geqq i$ signifies that $a \geqq i$ for every element $a$ of $\alpha$, and $\alpha<i$ that $a<i$ for every element $a$ of $\alpha$. The null sequence vacuously satisfies both $o \geqq i$ and $o<i$.

The sequence formed by adjoining $\beta$ to the end of $\alpha$ is denoted by $\alpha \beta$.

Definition. The relation $\beta \supset_{i} \alpha$ is to mean that a sequence $\alpha^{\prime}$ exists such that $\beta=\alpha \alpha^{\prime}$ and $\alpha^{\prime} \geqq i$.

Some immediate consequences of the preceding definitions are:

1.1. $\alpha \supset_{i} \alpha$.

1.2. If $\beta \supset_{j} \alpha$ and $j \geqq i$, then $\beta \supset_{i} \alpha$.

1.3. If $\gamma \supset_{i} \beta$ and $\beta \supset_{i} \alpha$, then $\gamma \supset_{i} \alpha$.

1.4. If $\gamma \supset_{a} \alpha$ and $\gamma \supset_{b} \beta$, then $\beta \supset_{a} \alpha$ or $\alpha \supset_{b} \beta$.

Proof. Let $\gamma \supset_{a} \alpha$ and $\gamma \supset_{b} \beta$; then sequences $\alpha^{\prime}, \beta^{\prime}$ exist such that

Presented to the Society, November 24, 1945; received by the editors August 24, 1945.

1 Paul Urysohn, Über die Mächtigkeit der zusammenhängenden Mengen, Math. Ann. vol. 94 (1925) pp. 262-295; see pp. 274-283 for the example. 
$\gamma=\alpha \alpha^{\prime}, \alpha^{\prime} \geqq a$, and $\gamma=\beta \beta^{\prime}, \beta^{\prime} \geqq b$. Since $\alpha \alpha^{\prime}=\beta \beta^{\prime}$, there exists a sequence $\alpha^{\prime \prime}$ such that $\beta=\alpha \alpha^{\prime \prime}$ or a sequence $\beta^{\prime \prime}$ such that $\alpha=\beta \beta^{\prime \prime}$. If $\beta=\alpha \alpha^{\prime \prime}$, then $\alpha \alpha^{\prime}=\beta \beta^{\prime}=\alpha \alpha^{\prime \prime} \beta^{\prime}$; hence $\alpha^{\prime}=\alpha^{\prime \prime} \beta^{\prime}$. But $\alpha^{\prime} \geqq a$, so $\alpha^{\prime \prime} \geqq a$ and consequently $\beta \supset_{a} \alpha$. Similarly, if $\alpha=\beta \beta^{\prime \prime}$, then $\alpha \supset_{b} \beta$.

2. Points in $X$. The space $X$ shall consist of two disjoint subsets: $Y$, the set of all even sequences; and $Z$, the set of all ordered pairs $\{k,(\mu, \nu)\}$ composed of a positive integer $k$ and a set $(\mu, \nu)=(\nu, \mu)$ of even sequences $\mu$ and $\nu$. Hereafter a point $\mu$ in $Y$ will be denoted by $y(\mu)$ and a point $\{k,(\mu, \nu)\}$ in $Z$ by $z_{k}(\mu, \nu)$. Evidently $X$ is countable.

The neighborhoods in $X$ will be formed from certain subsets $Y_{i}(\alpha)$ of $Y$, defined for every positive integer $i$ and every sequence $\alpha$.

Definition. $Y_{i}(\alpha)$ is the set of all points $y(\mu)$ such that $\mu \supset_{i} \alpha$.

Some properties of these sets are:

2.1. $y(\mu) \in Y_{i}(\mu)$.

2.2. If $j \geqq i$, then $Y_{j}(\alpha) \subset Y_{i}(\alpha)$.

2.3. If $y(\mu) \in Y_{i}(\alpha)$, then $Y_{i}(\mu) \subset Y_{i}(\alpha)$.

2.4. $Y_{a}(\alpha) Y_{b}(\beta) \neq 0$ is equivalent to: $\beta \supset_{a} \alpha$ or $\alpha \supset_{b} \beta$.

PRoof. If the set $Y_{a}(\alpha) Y_{b}(\beta)$ contains a point $y(\mu)$, then $\mu \supset_{a} \alpha$ and $\mu \supset_{b} \beta$; therefore, $\beta \supset_{a} \alpha$ or $\alpha \supset_{b} \beta$.

Now, if $\beta \supset_{a} \alpha$, define $m=\max (a, b), \nu=\beta$ if $\beta$ is even, $\nu=\beta m$ if $\beta$ is odd. Thus $\nu$ is even and $\nu \supset_{b} \beta$ so $y(\nu) \in Y_{b}(\beta)$. Moreover $\nu \supset_{a} \beta \supset_{a} \alpha$, hence $\nu \supset_{a} \alpha$ so $y(\nu) \in Y_{a}(\alpha)$. Therefore $Y_{a}(\alpha) Y_{b}(\beta) \neq 0$. Similarly $Y_{a}(\alpha) Y_{b}(\beta) \neq 0$ if $\alpha \supset_{b} \beta$.

Corollary. If $\alpha \neq \beta$ and $\alpha \beta<i$, then $Y_{i}(\alpha) Y_{i}(\beta)=0$.

To every point $z=z_{k}(\mu, \nu)$ a unique positive integer $q(z)=q_{k}(\mu, \nu)$ is assigned as follows. The set of all sets $(\mu, \nu)$ of even sequences $\mu$ and $\nu$, being countable and infinite, can be mapped onto the set of positive integral primes by some 1-1 mapping $p(\mu, \nu)$. Define

$$
q_{k}(\mu, \nu)=[p(\mu, \nu)]^{k}
$$

According to the unique factorization theorem of arithmetic, $q$ is a 1-1 mapping of the point set $Z$ onto a subset of the positive integers. Moreover, since the infinite sequence of positive integers $q_{k}(\mu, \nu)$ for $k=1,2, \cdots$ is strictly increasing, $q_{k}(\mu, \nu) \rightarrow \infty$ as $k \rightarrow \infty$. 
3. Neighborhoods in $X$. For every point $x$ in $X$ and every positive integer $i$, a neighborhood $V_{i} x$ of $x$ is now defined.

DEFinition.

$$
\begin{aligned}
V_{i} y(\mu) & =Y_{i}(\mu) \\
V_{i} z_{k}(\mu, \nu) & =z_{k}(\mu, \nu)+Y_{i}(\mu q)+Y_{i}(\nu q), \quad q=q_{k}(\mu, \nu) .
\end{aligned}
$$

Under this definition of neighborhood $X$ forms a Hausdorff topological space; that is, $X$ satisfies the following neighborhood axioms.

Axiom 1. To every point $x$ in $X$ there corresponds at least one neighborhood of $x$; every neighborhood of $x$ contains $x$ by 2.1 or by definition.

Axiom 2. If $V_{i} x$ and $V_{j} x$ are two neighborhoods of $x$, a neighborhood $V_{m} x$ of $x$ exists such that $V_{m} x \subset V_{i} x V_{j} x$. Indeed, if $m=\max (i, j)$, then $V_{m} x=V_{j} x V_{j} x$ by 2.2 .

Axiom 3. If $V_{i} x$ contains a point $y(\mu)$, there exists a neighborhood of $y(\mu)$ contained in $V_{i} x$. By 2.3 such a neighborhood is $V_{i} y(\mu)$.

Axiom 4H. Every two distinct points $x, x^{\prime}$ in $X$ are Hausdorff-or $H$-separable; that is, there exist neighborhoods $V_{i} x$ of $x$ and $V_{i} x^{\prime}$ of $x^{\prime}$ such that $V_{i} x V_{i} x^{\prime}=0$. The intersection $V_{i} x V_{i} x^{\prime}$ can be reduced to the sum of at most four intersections, each of the form $Y_{i}(\alpha) Y_{i}\left(\alpha^{\prime}\right)$. If $\alpha, \alpha^{\prime}$ are both even, then $\alpha \neq \alpha^{\prime}$ since $x \neq x^{\prime}$. And also $\alpha \neq \alpha^{\prime}$, if $\alpha, \alpha^{\prime}$ are both odd; for then even sequences $\mu, \mu^{\prime}$ and positive integers $q, q^{\prime}$ exist such that $\alpha=\mu q, \alpha^{\prime}=\mu^{\prime} q^{\prime}$, and, since $x \neq x^{\prime}, q \neq q^{\prime}$. Thus, according to the corollary of $2.4, Y_{i}(\alpha) Y_{i}\left(\alpha^{\prime}\right)=0$ when $i$ is chosen so that $\alpha \alpha^{\prime}<i$. An integer $i$ then exists for which $V_{i} x V_{i} x^{\prime}=0$.

Thus $X$ is a nondegenerate countable Hausdorff space. Evidently $X$ is also perfectly separable.

4. Connectedness of $X$. Two distinct points $x, x^{\prime}$ in a space $E$ are said to be $\bar{H}$-separable provided neighborhoods $V$ of $x$ and $V^{\prime}$ of $x^{\prime}$ exist such that $\bar{V} \bar{V}^{\prime}=0$; otherwise, the points $x, x^{\prime}$ are said to be $\bar{H}$-inseparable. A single point is also said to be $\bar{H}$-inseparable if it is $\bar{H}$-inseparable with every other point in $E$.

$A$ space $E$ containing an $\bar{H}$-inseparable point $x$ is connected; for otherwise $E$ could be covered by two non-null disjoint isolated (open and closed) sets $V, V^{\prime}$, one of which contains $x$; but this would imply the contradiction

$$
0=V V^{\prime}=\bar{V} \bar{V}^{\prime} \neq 0 .
$$

Moreover, if $E$ is a Hausdorff space, then no point of $E$ satisfies the regularity axiom, or, more briefly, is regular. For let $x^{\prime}$ be any point 
in $E$ distinct from $x$. Since $x, x^{\prime}$ are $H$-separable in $E$, there exist disjoint neighborhoods $V$ of $x$ and $V^{\prime}$ of $x^{\prime}$; consequently

$$
V \bar{V}^{\prime}=0=\bar{V} V^{\prime} \text {. }
$$

If $x$ were a regular point of $E$, then a neighborhood $U$ of $x$ would exist such that $V \supset \bar{U}$, so

$$
0=V \bar{V}^{\prime} \supset \bar{U} \bar{V}^{\prime} \neq 0 .
$$

Similarly, if $x^{\prime}$ were a regular point of $E$, then a neighborhood $U^{\prime}$ of $x^{\prime}$ would exist such that $V^{\prime} \supset \bar{U}^{\prime}$, so

$$
0=\bar{V} V^{\prime} \supset \bar{V} \bar{U}^{\prime} \neq 0 .
$$

By considering the sets $Y_{i}(\alpha)$ every point in the space $X$ is now shown to be $\bar{H}$-inseparable. Hence $X$ is connected and no point of $X$ is regular.

Definition. $Z_{i}(\alpha)$ is the set of all points $z_{k}(\mu, \nu)$ such that $\mu q \supset_{i} \alpha$ or $\nu q \supset_{i} \alpha, q=q_{k}(\mu, \nu)$.

4.1. $\bar{Y}_{i}(\alpha)=Y_{i}(\alpha)+Z_{i}(\alpha)$.

Proof. The following equivalent statements show that $Y \bar{Y}_{i}(\alpha)$ $=Y_{i}(\alpha)$ :

$y(\mu) \in \bar{Y}_{i}(\alpha)$.

For all $j: V_{j} y(\mu) Y_{i}(\alpha) \neq 0$.

For all $j: Y_{j}(\mu) Y_{i}(\alpha) \neq 0$.

For all $j: \alpha \supset_{j} \mu$ or $\mu \supset_{i} \alpha$.

$\mu \supset_{i} \alpha$.

$y(\mu) \in Y_{i}(\alpha)$.

The following equivalent statements show that $Z \bar{Y}_{i}(\alpha)=Z_{i}(\alpha)$, where $q_{k}(\mu, \nu)$ has been abbreviated to $q$ :

$z_{k}(\mu, \nu) \in \bar{Y}_{i}(\alpha)$.

For all $j: V_{j} z_{k}(\mu, \nu) Y_{i}(\alpha) \neq 0$.

For all $j:\left[Y_{j}(\mu q)+Y_{j}(\nu q)\right] Y_{i}(\alpha) \neq 0$.

For all $j: Y_{i}(\mu q) Y_{i}(\alpha) \neq 0$ or $Y_{i}(\nu q) Y_{i}(\alpha) \neq 0$.

For all $j: \alpha \supset_{j} \mu q$ or $\mu q \supset_{i} \alpha$ or $\alpha \supset_{j} \nu q$ or $\nu q \supset_{i} \alpha$.

$\mu q \supset_{i} \alpha$ or $\nu q \supset_{i} \alpha$.

$z_{k}(\mu, \nu) \in Z_{i}(\alpha)$.

4.2. $Z_{a}(\alpha) Z_{b}(\beta) \neq 0$; hence every two distinct points in $X$ are $\bar{H}$-inseparable.

Proof. Evidently there exist even sequences $\mu, \nu$ such that $\mu \supset_{a} \alpha$ and $\nu \supset_{b} \beta$. And since $q_{k}(\mu, \nu) \rightarrow \infty$ as $k \rightarrow \infty$ a positive integer $k$ exists for which 


$$
q=q_{k}(\mu, \nu) \geqq \max (a, b) .
$$

Therefore $\mu q \supset_{a} \alpha$ and $\nu q \supset_{b} \beta$; so $z_{k}(\mu, \nu) \in Z_{a}(\alpha) Z_{b}(\beta)$.

Thus $X$ is an $\Im$-space whose every point is $\bar{H}$-inseparable.

5. The space $X^{*}$. Let $X^{*}$ be the relative subspace of $X$ formed by removing from $X$ all points $z_{k}(\mu, \nu)$ except those of the form $z_{k}(\mu, o)$, $\mu \neq 0$. Notice that every $X^{*}$-neighborhood of a point in $X^{*}$ is also an $X$-neighborhood of that point. The argument of 4.2 shows that the set $Z_{a}(\alpha) Z_{b}(0)$ contains a point of $X^{*}$. The point $y(0)$ is then an $\bar{H}$-inseparable point of $X^{*}$. Thus $X^{*}$, being a nondegenerate connected subspace of an S-space, is also an S-space.

6. The space $X^{* *}$. Let $X^{* *}$ be the relative subspace of $X^{*}$ formed by removing from $X^{*}$ the single point $y(o)$. This point is a dispersion point of $X^{*}$; for the following recursive construction of isolated subsets in the space $X^{* *}$ shows that $X^{* *}$ is totally disconnected.

Definition. For every non-null even sequence $\lambda$ and every positive integer $i$ such that $\lambda<i$ let

$$
X_{i}(\lambda)=\sum_{n=1}^{\infty}\left[Y_{i}^{n}(\lambda)+Z_{i}^{n}(\lambda)\right]
$$

the sets $Y_{i}^{n}(\lambda)$ and $Z_{i}^{n}(\lambda)$ being recursively defined as follows:

$Y_{i}^{n}(\lambda)$ is the set of all points $y(\mu)$ such that $\mu \supset, \alpha^{n}$, where $\alpha^{n}=\lambda$ if $n=1$, and $\alpha^{n}=q(z)$ for some $z \in Z_{i}^{n-1}(\lambda)$ if $n>1$;

$Z_{i}^{n}(\lambda)$ is the set of all points $z=z_{k}(\mu, 0)$ such that $y(\mu) \in Y_{i}^{n}(\lambda)$ and $q(z) \geqq i$.

6.1. $V_{i} x \subset X_{i}(\lambda)$ for all $x \in X_{i}(\lambda)$; hence $X_{i}(\lambda)$ is open in $X^{* *}$.

Proof. Let $y(\mu) \in Y_{i}^{n}(\lambda)$; then $\mu \supset, \alpha^{n}$. If $y(\nu) \in V_{i} y(\mu)$, then $\nu \supset_{\imath} \mu \supset_{i} \alpha^{n}$, so $y(\nu) \in Y_{i}^{n}(\lambda)$.

Let $z=z_{k}(\mu, o) \in Z_{i}^{n}(\lambda)$; then $\mu \supset_{i} \alpha^{n}$ and $q(z) \geqq i$. If $y(\nu) \in V_{i} z$, then $\nu \supset_{i} \mu q(z)$ or $\nu \supset_{i} q(z)$. Now $\nu \supset_{i} \mu q(z), q(z) \geqq i$, implies that $\nu \supset_{i} \mu \supset_{i} \alpha^{n}$ and hence that $y(\nu) \in Y_{i}^{n}(\lambda)$. And $\nu \supset_{i} q(z), z \in Z_{i}^{n}(\lambda)$, implies that $y(\nu) \in Y_{i}^{n+1}(\lambda)$.

\section{2. $V_{i} x X_{i}(\lambda)=0$ for all $x \in X_{i}(\lambda)$; hence $X_{i}(\lambda)$ is closed in $X^{* *}$.}

Proof. Let $y(\mu) \notin X_{i}(\lambda)$. Suppose the set $V_{i} y(\mu) X_{i}(\lambda)$ contains a point $y(\nu)$; then $\nu \supset_{\imath} \mu$ and $\nu \supset_{\imath} \alpha^{n}$. Therefore $\mu \supset_{i} \alpha^{n}$ or $\alpha^{n} \supset_{\imath} \mu$. Now $\alpha^{n} D_{2} \mu$, since $\alpha^{1}=\lambda<i$ and since $\alpha^{n}$ is a single integer if $n>1$. Hence $\mu \supset$; $\alpha^{n}$, so $y(\mu) \in Y_{i}^{n}(\lambda)$-a contradiction.

Let $z=z_{k}(\mu, 0) \notin X_{i}(\lambda)$. Suppose the set $V_{i} z X_{i}(\lambda)$ contains a point 
$y(\nu)$; then $\nu \supset_{i} \mu q(z)$ or $\nu \supset_{i} q(z)$, and $\nu_{\supset_{i}} \alpha^{n}$. Therefore

$$
\mathfrak{P}^{n}: \alpha^{n} \supset_{i} \mu q(z) \text { or } \mu q(z) \supset_{i} \alpha^{n} \text { or } \alpha^{n} \supset_{i} q(z) \text { or } q(z) \supset_{i} \alpha^{n} \text {. }
$$

Now $\lambda \neq 0, \lambda<i$, and $\lambda=\alpha^{1} ; \mathfrak{A}^{1}$ then reduces to $\mu q(z) \supset_{i} \lambda$; so $\mu_{\supset_{i} \lambda}$, $q(z) \geqq i$, and consequently $z \in Z_{i}^{1}(\lambda)$-a contradiction. If $n>1$, then $\alpha^{n}=q\left(z^{\prime}\right) \geqq i$ for some $z^{\prime} \in Z_{i}^{n-1}(\lambda)$, so $\mathfrak{A}^{n}$ reduces to: $\mu q(z) \supset_{i} q\left(z^{\prime}\right)$, $\mu \neq 0$; or $q(z)=q\left(z^{\prime}\right)$. Now $\mu q(z) \supset_{i} q\left(z^{\prime}\right), \mu \neq 0$, implies that $\mu \supset_{i} q\left(z^{\prime}\right)$, $q(z) \geqq i$, and hence that $z \in Z_{i}^{n}(\lambda)$-a contradiction. And $q(z)=q\left(z^{\prime}\right)$ implies that $z=z^{\prime} \in Z_{i}^{n-1}(\lambda)$-also a contradiction.

The sets $X_{i}(\lambda)$ are then isolated subsets of $X^{* *}$ for $\lambda \neq 0, \lambda<i$. Notice that

$$
\begin{aligned}
x=y(\lambda) \in X_{i}(\lambda), & \\
x=y\left(\lambda^{\prime}\right) \notin X_{i}(\lambda) \quad \text { if } & \lambda^{\prime} \neq \lambda \text { and } \lambda^{\prime}<i, \\
x=z_{k}(\lambda, o) \in X_{i}(\lambda) & \text { if } \quad q(x) \geqq i, \\
x^{\prime} \notin X_{i}(\lambda) & \text { if } \quad x^{\prime} \in Z \text { and } q\left(x^{\prime}\right)<i .
\end{aligned}
$$

Now there exists for any two distinct points $x, x^{\prime}$ in $X^{* *}$ an isolated set $X_{i}(\lambda)$ containing $x$ but not $x^{\prime}$ : if $x=y(\lambda), x^{\prime}=y\left(\lambda^{\prime}\right)$, choose $i$ so that $\lambda \lambda^{\prime}<i$; if $x=y(\lambda)$ and $x^{\prime} \in Z$, choose $i$ so that $\lambda q\left(x^{\prime}\right)<i$; and if $x=z_{k}(\lambda, 0)$ and $x^{\prime} \in Z$, choose $i=q(x)$, then $q\left(x^{\prime}\right)<i$ and $\lambda<i$, since it may be assumed that $q\left(x^{\prime}\right)<q(x)$ and since the mapping $p$ can. be selected so that $\mu \nu<p(\mu, \nu)$.

Thus the space $X^{* *}$ is totally disconnected. In particular, every two distinct points in $X^{* *}$ are $\bar{H}$-separable; hence $y(0)$ is the only $\bar{H}$-inseparable point of $X^{*}$.

University of California at Los Angeles 